\title{
Numerical Study of Wall Shear and Velocities Using a Commercial CFD Code: Some Crucial Aspects to Consider
}

Binaya Baidar, Michel J. Cervantes

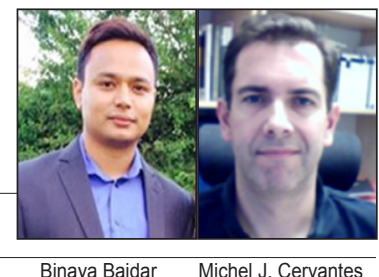

Abstract: This paper presents the numerical prediction of wall shear and velocities in steady and superposed pulsatile turbulent flow in a pipe, the phenomena that can be observed in hydropower. The previously conducted experiment is a base for this study and some crucial aspects of CFD while using a commercial code have been emphasized. The widely-accepted grid convergence index approach is adopted to quantify the discretization uncertainty and the results are validated against the experiment. The influence of the wall functions applied in the code is also studied with two turbulence models: standard $k-\varepsilon$ and $k \omega$ based SST model. The time-averaged results of superposed flow with small amplitude unsteadiness are equivalent to results from the steady flow. The results and the method used in this paper may be useful for the CFD simulations in hydropower applications like penstock and bifurcations designs.

Keywords: Law of the wall, wall shear, velocity, turbulent flow, CFD

\section{Introduction}

Computational Fluid Dynamics (CFD) has now been a ${ }_{\text {well-established technique in hydropower research }}$ and development. Increasing number of publications and research based on CFD are currently seen in the hydropower applications (Trivedi, et al., 2016). The quality and trust in the CFD results are utmost important in relying on its results. The CFD simulations can have several sources of error which can be, in most cases, difficult to get identified. The common sources of error, assuming the correct use of the software and the software itself is correct, are the numerical errors and modeling errors. As the CFD relies on the discretization of the partial differential equations of the mass, momentum and energy equations (commonly known as the NavierStokes equation), there is always some difference in the exact solution and the approximate solution estimated by CFD. These differences are attributed to numerical errors. Whereas, the modelling errors result from the use of some empirical models implemented in a software that describes flow behavior, like turbulence or the wall function.

The purpose of using the wall function in CFD is to lower the computational effort with the acceptable meshing. This approach uses the law of the wall and the first computational node is placed outside the viscous sublayer of the boundary layer. The mean velocity is proportional to the $\log$ from the wall distance. Then the near wall velocity profile is assumed to obtain the wall shear stress from the effect of the outer flow conditions. Here the mean velocity $U$ is assumed to be only the function of wall shear $\tau \omega$, distance from the wall surface $y$, the fluid density $\rho$, and kinematic viscosity $v$. It can be presented in the dimensionless form as $U^{\wedge}+=U / \ell_{-} \tau$ $=f\left(\left(u \_\tau y\right) v\right)=f\left(y^{\wedge}+\right)$ (Kundu, et al., 2015). And it can be presented as

$$
U^{+}=U / u_{\tau}=f\left(y^{+}\right)=\frac{1}{k} \ln \left(y^{+}\right)+C
$$

$k$ is the von Karman constant and the experimental $\mathrm{v}$ a $\mathrm{l} \mathrm{u} \mathrm{e} \sim$ is $\mathrm{k} 0.4$ and is around 4 or 5 for a smooth $\mathrm{w}$ a $1 \mathrm{l}$. The term $y^{+}$is given by $\left(u \_\tau y\right) / v$, where $u_{\tau}$ is the frictional velocity given by $u \tau=\sqrt{ }(\tau \omega / \rho)$. The Eqn. (1) is called the law of the wall. The wall function approach in CFD uses this empirical method to impose suitable conditions without resolving the boundary layer. However, this method is insufficient to understand the boundary layer separation or rotating fluid. Therefore, if we are interested in boundary layer phenomena, this approach may not be that accurate.

This paper presents the crucial aspects of utilizing a commercial code by studying the wall shear and velocity in a turbulent flow, which may be useful to the CFD users of Nepal. In this regard, a numerical model resembling the previously conducted experiment in a pipe has been developed and used as the test case. Some experimental conditions: both steady and pulsatile superposed flow, were applied in the study. The study of the wall shear and velocity profiles can also be useful in the other areas of the study, for instances, the Pressure time method in discharge measurement or in designing penstocks and bifurcations.

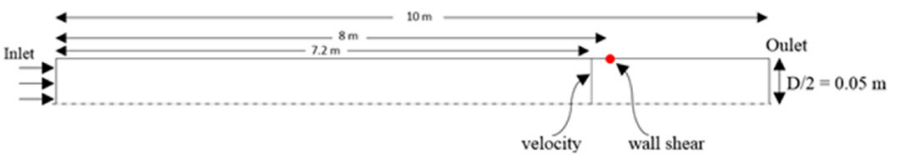

Figure. 1: Domain information and the test location of velocity and the wall shear stress. Velocity measurement was done at $7.2 \mathrm{~m}$ from the inlet and the wall shear measurement was done at $8 \mathrm{~m}$ from the inlet. The total length of the pipe is $10 \mathrm{~m}$ and its diameter is $0.1 \mathrm{~m}$.

\section{Method}

The test case of Sundström et al. (2016) was used in this study. The schematic diagram is shown in Fig. 1. The measurement locations for velocity and wall shear stress were at $7.2 \mathrm{~m}$ and $8 \mathrm{~m}$ respectively from the inlet. The 
velocity was measured with the help of a two-component LDV system from Dantec Dynamics and the wall shear was measured with a flush-mounted (55R46) hot-film probe.

The mean Reynolds number $R e=U_{b} D / v$ was 14500 within $0.4 \%$ during the experiment, where $D$ is the pipe diameter. The Karman number $\operatorname{Re}_{\tau}=\mu \tau D / v$ during the experiment was 900 within $1 \%$, so the frictional velocity, $\mu \tau$, is in average equal to $0.009036 \mathrm{~m} / \mathrm{s}$.

\section{Computational Model}

The commercial CFD code ANSYS CFX v16.o was used for the numerical studies. The grid was created in ICEM CFD with a single element thickness. Four sets of grids were generated: a) coarsest grid $\mathrm{G}_{4}$ without resolving the boundary layer $\left(y^{+} \sim 22\right), 13980$ cells, b) coarse grid $\mathrm{G} 3$ without resolving the viscous sublayer $\left(y^{+} \sim 5\right), 23067$ cells, c) medium grid G2 with resolved boundary layer (y+ O.7), 169387 cells and d) fine grid G1 with fully resolved boundary layer $(y+\sim O .3), 429141$ cells. The node incremental ratio was kept at 1.15.

The axisymmetric condition was established with the no-slip condition at the wall was employed. The inlet velocity was prescribed to match the Reynolds number of the experiment and the outlet was considered as the fully developed flow by prescribing the static pressure. The high-resolution scheme of the code was selected for the spatial discretization. This scheme is the blend of the first and second order to be stable. It also assures the accuracy to be as close as possible to a second orde. (ANSYS, 2016). The turbulence numerics were chosen as the first order. The RMS residual in the mass and momentum equations was set to $1 \mathrm{E}-7$ and the variables to reach the stable state as the convergence control. The unsteady simulations were also carried out with the above-mentioned settings and the transient scheme was chosen as the second order backward Euler.

Two turbulence models were studied: $k-\varepsilon$ standard with the wall function and Menter's $k \omega$ based Shear Stress Transport (Menter, 1994). In the CFX code, the $k-\varepsilon$ model utilizes the scalable wall function, which virtually moves the first computational node to be outside the viscous sublayer. while the SST model automatically switches to the wall function approach from the low$R e$ formulation if the grid is not refined till the viscous sublayer.

\section{Estimation of Discretization Error}

The widely-accepted Richardson extrapolation method was used to estimate discretization error. The method is well described in (Celik, et al., 2008) and briefly mentioned here and followed accordingly. For a representative cell size $h$ and considering $h_{1}<h_{2}<h_{3}$, the apparent order, $p$, of convergence is given by the expressions
Where denotes the solution on the $i^{\text {th }}$ grid and $r_{21}=h_{2} / h_{1}$

$$
\begin{aligned}
p= & \frac{1}{\ln \left(r_{21}\right)}|\ln | \varepsilon_{32} / \varepsilon_{21}|+q(p)|, \\
& q(p)=\ln \left(\frac{r_{21}^{p}-s}{r_{32}^{p}-s}\right), \\
& s=1 \cdot \operatorname{sign}\left(\varepsilon_{32} / \varepsilon_{21}\right) \\
, r_{32}= & h_{3} \varepsilon_{32}=\emptyset_{3}-\emptyset_{2,} \varepsilon_{21}=\emptyset_{2}-\emptyset_{1}, \emptyset_{i} / h_{2} .
\end{aligned}
$$

Then the approximate relative error is

$$
e_{a}^{21}=\left|\frac{\emptyset_{1}-\emptyset_{2}}{\emptyset_{1}}\right|
$$

The extrapolated values are given by:

$$
\emptyset_{\text {ext }}^{21}=\frac{\left(r_{21}^{p} \emptyset_{1}-\emptyset_{2}\right)}{\left(r_{21}^{p}-1\right)}, \text { Similarly, for } \emptyset_{\text {ext }}^{32}
$$

The fine grid convergence index is then

$$
\text { GCI }_{\text {fine }}^{21}=\left|\frac{1.25 e_{a}^{21}}{r_{21}^{p}-1}\right|
$$

The discretization error and uncertainties computed applying the above method in the present analysis is resented in Table 1.

\begin{tabular}{|l|l|}
\hline Parameter & \multicolumn{1}{|c|}{$\begin{array}{l}\text { Wall shear stress } \\
(\mathbf{P a}) \text { at } \mathbf{x} / \mathbf{L}=\mathbf{0 . 8}\end{array}$} \\
\hline$r_{-21}$ & 1.36 \\
\hline$r_{-32}$ & 1.94 \\
\hline$p$ & 0.63 \\
\hline $\mathrm{GCI}^{21}$ fine & $7.88 \%$ \\
\hline $\mathrm{GCI}^{32}{ }_{\text {med }}$ & $4.58 \%$ \\
\hline
\end{tabular}

Table 1: Discretization error and uncertainties in the numerical solutions

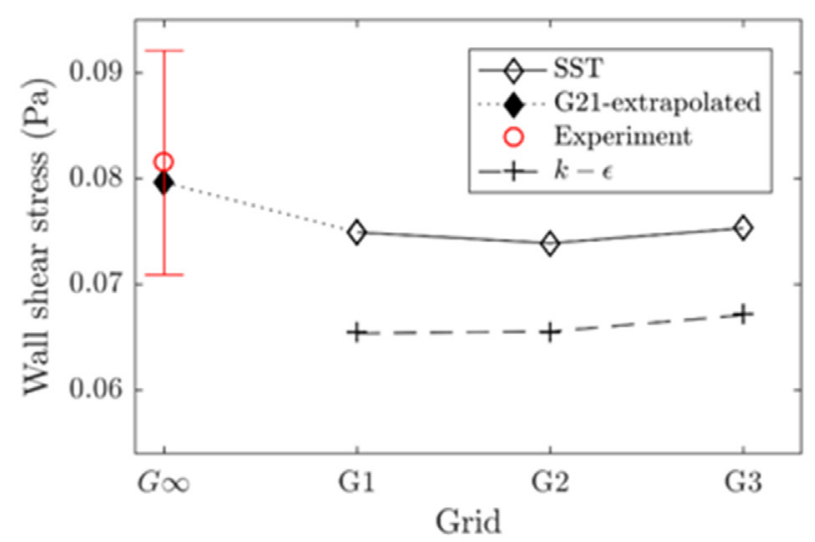

Figure 2: Wall shear stress for different grids with standard $k-\varepsilon$ and SST turbulence model. The extrapolation is done per the $\mathrm{GCl}$ method and $\mathrm{G}_{\infty}$ means if the number of cells in the grid reach infinity. The wall shear predicted by the SST model for all the grids are in good agreement with experimental data than that predicted by the $k-\varepsilon$ model. The uncertainty in the experiment was 13. (Sundstrom, et al., 2016). 
The estimated numerical uncertainties for the wall shear stress at $8 \mathrm{~m}$ from the inlet is $7.88 \%$ and $4.58 \%$ error using the fine grid from the medium grid, respectively.

\section{Results and Discussions Steady results}

The wall shear stress for the grid types: G1, G2 and G3, and the both turbulence models considered are shown in Fig. 2. The wall shear predicted by the SST turbulence model is in good agreement with the experiment. The extrapolated wall shear shows very good match with the experiment; error below $2.5 \%$. The extrapolated value means if the grid was resolved infinitely (Go in Fig. 2), the solution is exact.

For the fine grid (G1), the model failed to converge to the prescribed condition, as this model has problems in numerical instability while handling the low turbulent Reynolds number conditions. The closest wall shear predicted by this model, with the G3 grid, has a discrepancy of $18 \%$ compared to the experimental result.

The velocity profile at the line, which $7.2 \mathrm{~m}$ from the inlet of the domain (shown in Fig. 1) for all the grids and both turbulence models are shown in Fig. 3 a-d. The wall velocities are expressed in the wall units, i.e. $U^{+}$versus $Y^{+}$. The curve $U^{+}=13.3 \tanh \left(\mathrm{y}^{+} / 13.3\right)$ and the log-law are also plotted in the figures. For all the grids, the velocity is well predicted in the log-law region either the model is using or without using the wall function.

As the boundary layer is fully resolved in the G1 and G2 grids, the viscous sublayer is well captured from the simulations using the SST turbulence model (Fig. 3 a-b). However, the results for $k-\varepsilon$ does not capture the viscous sublayer because the code uses the scalable wall functions, which virtually puts the first computational node outside the viscous sublayer. The velocity profile for the grid G1 is shown in Fig. 4, where the effect of the scalable wall function is clearly seen for $k-\varepsilon$ turbulence model. The first computation node is placed outside the viscous sublayer.

The G3 grid also shows the similar behavior like G1 and G2 grid (Fig. 3c), but it does not resolve the viscous sublayer. The grid G4 uses the wall function for both the grids, which accurately predicts the velocity in the log-law region Fig. 3 d. The $U^{+}$values are slightly overpredicted while using $k-\varepsilon$ model in both cases.

The turbulent axial velocity fluctuation ${\overline{u^{\prime}}}^{+}$from $\quad$ G1 using SST turbulence model is compared with the experimental data in Fig. 5. The result of the velocity fluctuation $u^{\prime}$ was calculated from the Boussines eddy viscosity approximation. This assumption can be expressed in the following form for the Reynolds stress tensor $-\rho \overline{u_{\imath}^{\prime} u_{j}^{\prime}}$ as: $-\rho \overline{u_{\imath}^{\prime} u_{\jmath}^{\prime}}=\mu_{t}\left(\frac{\partial U_{i}}{\partial x_{j}}+\frac{\partial U_{j}}{\partial x_{i}}\right)-\frac{2}{3} \rho k \delta_{i j}$

Where $\mu_{t}$ is eddy viscosity, $K$ is the turbulent kinetic energy, $\delta_{i j}$ is Kronecker delta function. The velocity fluctuation is not seen to be captured enough with the SST model.
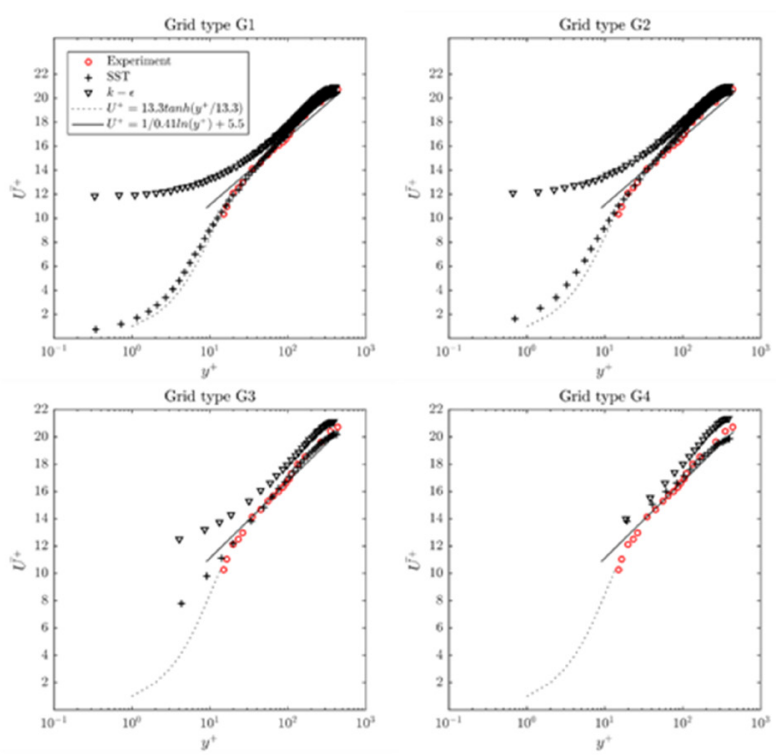

Figure 3: $U^{+}$versus $y^{+}$for all four sets of grids to study the near wall treatment. The numerical results for all grids are in good agreement with the law of the wall and the experiment in the log-law region for both turbulence models: $k-\varepsilon$ and SST. The influence of using the scalable wall function in the code is seen for the $k-\varepsilon$ in $\mathrm{G} 1$ and G2. The viscous sublayer is well predicted while using low-Re formulation, i.e. in SST turbulence model while the boundary layer is fully resolved G1 and G2. The experimental results are from (Sundstrom, et al., 2016).

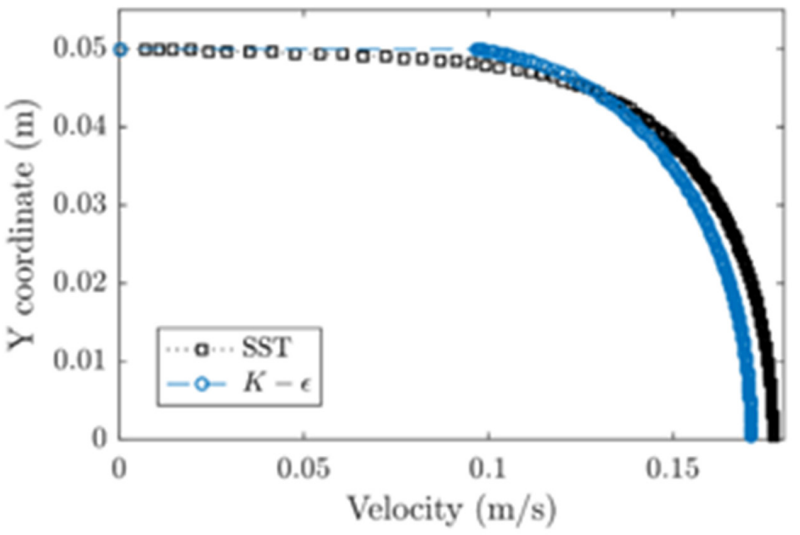

Figure 4: Velocity profile for the fine grid (G1) using SST and $k-\varepsilon$ turbulence models. The scalable wall function approach for $k-\varepsilon$ places the first computational node outside the viscous sublayer, unlike with the SST. Note that the first node is always on the wall. The $Y$ coordinate is from the center of the pipe, so the wall is at $0.05 \mathrm{~m}$. 


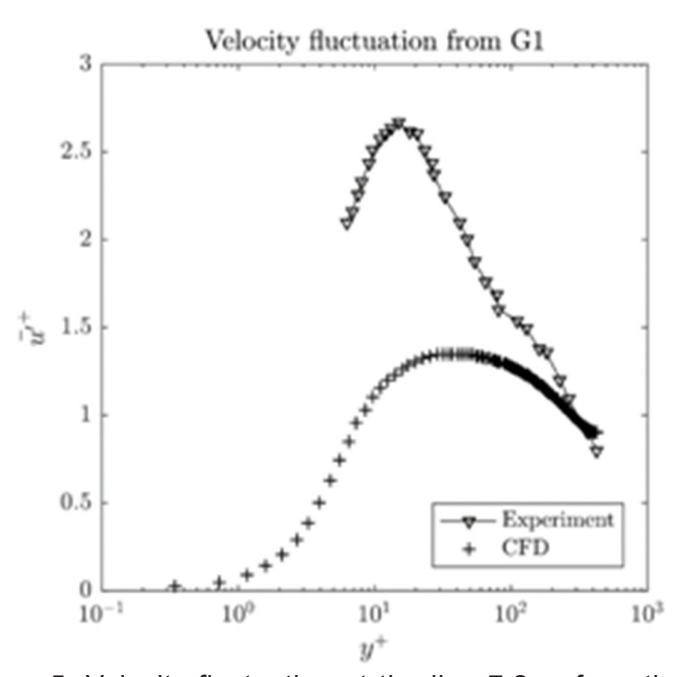

Figure 5: Velocity fluctuation at the line $7.2 \mathrm{~m}$ from the inlet, shown in Fig.1 with the fine grid G1 assuming the eddy viscosity model. The fluctuations predicted by CFD is less that the experiment. The experimental results are from (Sundstrom, et al., 2016).

\section{Unsteady results}

The wall shear stress with single, double and superposed pulsating flow was investigated. The amplitude and frequency are in accordance with the experiment and hence compared here. As the timeaveraged result in the experiment is closely related with the steady one, the latter one is only mentioned here for comparison. Table 2 shows the investigated cases and its values. convergence study was done. The SST turbulence model with similar conditions as the previous simulations was considered here as well.

The time-averaged wall shear is at most $7 \%$ difference from the steady results, $\mathrm{L}_{\mathrm{S}}^{+}=25$ for (Fig. 6). Furthermore, the velocities are also similar with the steady results that were presented in Fig. 3a, see Fig. 7. It suggests that even in the pulsatile superposed flows, the steady simulations are enough to predict the time-averaged results.

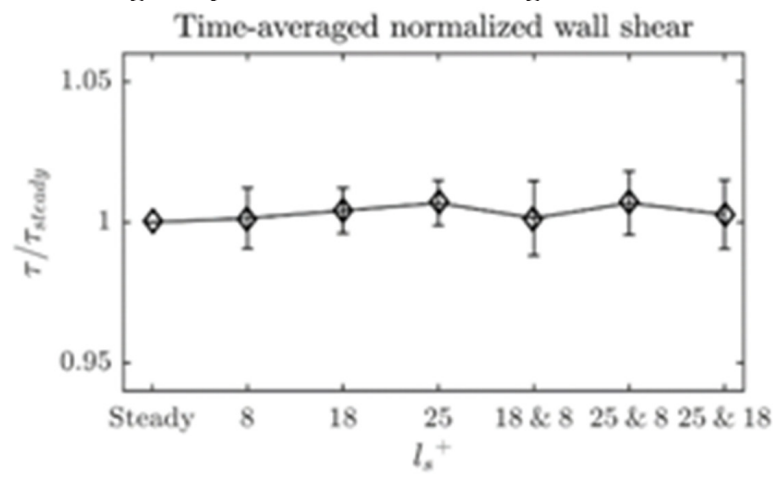

Figure 6: Normalized wall shear stress for the steady and unsteady condition with error bar representing the standard deviation.

\section{Summary}

The time-averaged numerical predictions of the wall shear and velocities are in good agreement with the steady results and experimental results, which signifies the steady simulation are sufficient if the averaged results

\begin{tabular}{|l|c|c|c|c|c|c|}
\hline & $\begin{array}{c}\text { Low fre- } \\
\text { quency } \\
\text { (LF) }\end{array}$ & $\begin{array}{c}\text { Intermediate } \\
\text { Freque n cy } \\
\text { (IF) }\end{array}$ & $\begin{array}{c}\text { Q u a s i } \\
\text { laminar } \\
\text { (QL) }\end{array}$ & LF \& IF & $\begin{array}{c}\text { LF \& } \\
\text { QL }\end{array}$ & $\begin{array}{c}\text { IF QL } \\
\text { QL }\end{array}$ \\
\hline$f(H z)$ & 0.04 & 0.08 & 0.4 & $\begin{array}{c}0.04 \& \\
0.08\end{array}$ & $\begin{array}{c}0.04 \& \\
0.4\end{array}$ & $\begin{array}{c}0.08 \quad \& \\
0.4\end{array}$ \\
\hline$l_{s}^{+}$ & 25 & 18 & 8 & $25 \& 18$ & $25 \& 8$ & $18 \& 8$ \\
\hline $\begin{array}{l}\text { Amplitude } \\
\text { (\% of bulk } \\
\text { flow rate) }\end{array}$ & 10 & 10 & 7.5 & $10 \& 10$ & 10 & $10 \& 7.5$ \\
\hline
\end{tabular}
are preferred. The velocity profiles are well-predicted by both turbulence models: and SST - low-Re formulation, using and without using the wall functions. However, the wall shear is more accurate with the SST turbulence model; with an error below $8 \%$, even though the uncertainty in the experimental result was $13 \%$. The least discrepancy achieved with the model was as high as $18 \%$ to the experiment. It was also seen that both the turbulence models

Table 2: Simulated condition according with the experiment in (Sundstrom, et al., 2016). $\mathrm{I}_{\mathrm{s}}^{+}$is the Stokes length normalized in wall units given by $\mathrm{I}_{s}^{+}=\sqrt{2} / \omega^{+}, \omega^{+}$is the angular forcing/ pulsating frequency scaled in wall units and given by $\omega^{+}=\omega u / u^{-}$ ${ }_{\mathrm{T}}^{2}$. Here $\mathrm{u}_{\mathrm{T}}^{-}$is the time averaged friction velocity.

For the unsteady simulation, the time step was chosen as the $0.125 \mathrm{~s}$ with the total time of $100 \mathrm{~s}$. The RMS and maximum Courant number were 2.8 and 11 respectively, which is allowable as the second order backward Euler scheme; implicit scheme and second order accurate, was used in the solution. The maximum frequency in the input was $0.4 \mathrm{~Hz}$ (for quasi-laminar QL case, see Table 2), so the time step is chosen as the 20 times smaller than the time period. Moreover, the purpose of this simulation was to time average the results, hence this time step is sufficient for the studies. Therefore, no temporal using and without using wall function cannot capture turbulence velocity fluctuations.
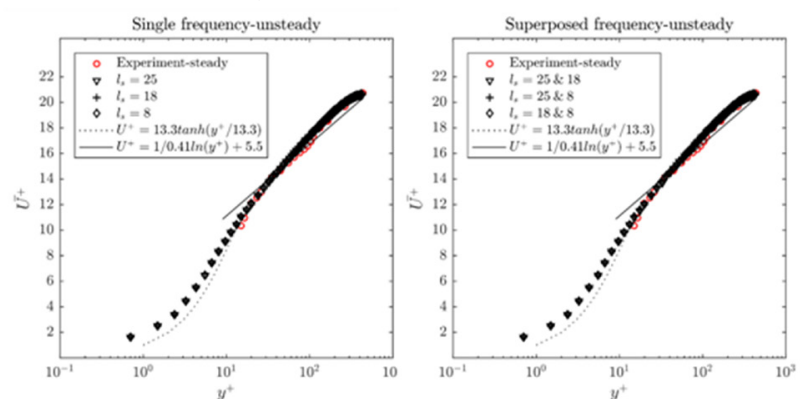

Figure 7: Single frequency (left) and superposed frequency (right) time-averaged results compared with the steady experimental results from (Sundstrom, et al., 2016). The time averaged results are in good agreement with the experiment. 
Binaya Baidar is studying for a Ph.D. at the Fluid Mechanics division in Lulea University of Technology, Sweden. He holds master's degree in Turbomachinery Aeromechanics (2013) from KTH (Sweden), University of Liege (Belgium) and Aristotle University (Greece). His current research is related to investigation of the Winter-Kennedy method in hydropower discharge measurement.

Michel Cervantes is a Professor at Luleå University of Technology, Sweden. He is also an adjunct professor at the Norwegian University of Science of Technology, Norway. He received his Ph.D. in 2003. His research focuses on hydropower, experimentally and numerically, more specifically concerned with the effects of unsteadiness on the losses, rotor-stator interaction, flow design and flow measurements techniques.

\section{References}

ANSYS, 2016. Ansys 16 CFX solver guide, s.l.: ANSYS.

Celik, I. et al., 2008. Procedure for Estimation and Reporting of Uncertainty due to Discretization in CFD Applications. ASME J. Fluids Eng., Volume 130, pp. 078001-(1-4).

Kundu, P., Cohen, I. \& Dowling, D., 2015. Fluid Mechanics. Sixth ed. s.l.:Academic Press.

Menter, F., 1994. Two-Equation Eddy-Viscosity Turbulence Models for Engineering Applications. AIAA Journal, 32(8), pp. 1598-1605.

Sundstrom, J. L. R., Mulu, B. G. \& Cervantes, M. J., 2016. Wall friction and velocity measurements in a doublefrequency pulsating turbulent flow. Journal of Fluid Mechanics, Volume 788, pp. 521-548.

Trivedi, C., Cervantes, M. J. \& Dahlhaug, O. G., 2016. Numerical Techniques Applied to Hydraulic Turbines: A Perspective Review. ASME Applied Mechanics Reviews, Volume 68, p. 010802. 